\title{
Stereoelectroencephalography for continuous two-dimensional cursor control in a brain-machine interface
}

\author{
Sumeet Vadera, M.D., ${ }^{1}$ Amar R. Marathe, Ph.D., ${ }^{2-5}$ \\ Jorge Gonzalez-Martinez, M.D., Ph.D., ${ }^{1}$ And Dawn M. Taylor, Ph.D. ${ }^{2,3,5}$ \\ Departments of ${ }^{1}$ Neurosurgery and ${ }^{2}$ Neurosciences, Cleveland Clinic; ${ }^{3}$ Department of Biomedical \\ Engineering, Case Western Reserve University; ${ }^{4}$ Cleveland Functional Electrical Stimulation (FES) \\ Center of Excellence, Louis Stokes VA Medical Center, Cleveland, Ohio; and ${ }^{5}$ Human Research and \\ Engineering Directorate, US Army Research Laboratory, Aberdeen Proving Ground, Maryland
}

\begin{abstract}
Stereoelectroencephalography (SEEG) is becoming more prevalent as a planning tool for surgical treatment of intractable epilepsy. Stereoelectroencephalography uses long, thin, cylindrical "depth" electrodes containing multiple recording contacts along each electrode's length. Each lead is inserted into the brain percutaneously. The advantage of SEEG is that the electrodes can easily target deeper brain structures that are inaccessible with subdural grid electrodes, and SEEG does not require a craniotomy. Brain-machine interface (BMI) research is also becoming more common in the Epilepsy Monitoring Unit. A brain-machine interface decodes a person's desired movement or action from the recorded brain activity and then uses the decoded brain activity to control an assistive device in real time. Although BMIs are primarily being developed for use by severely paralyzed individuals, epilepsy patients undergoing invasive brain monitoring provide an opportunity to test the effectiveness of different invasive recording electrodes for use in BMI systems. This study investigated the ability to use SEEG electrodes for control of 2D cursor velocity in a BMI. Two patients who were undergoing SEEG for intractable epilepsy participated in this study. Participants were instructed to wiggle or rest the hand contralateral to their SEEG electrodes to control the horizontal velocity of a cursor on a screen. Simultaneously they were instructed to wiggle or rest their feet to control the vertical component of cursor velocity. The BMI system was designed to detect power spectral changes associated with hand and foot activity and translate those spectral changes into horizontal and vertical cursor movements in real time. During testing, participants used their decoded SEEG signals to move the brain-controlled cursor to radial targets that appeared on the screen. Although power spectral information from 28 to 32 electrode contacts were used for cursor control during the experiment, post hoc analysis indicated that better control may have been possible using only a single SEEG depth electrode containing multiple recording contacts in both hand and foot cortical areas. These results suggest that the advantages of using SEEG for epilepsy monitoring may also apply to using SEEG electrodes in BMI systems. Specifically, SEEG electrodes can target deeper brain structures, such as foot motor cortex, and both hand and foot areas can be targeted with a single SEEG electrode implanted percutaneously. Therefore, SEEG electrodes may be an attractive option for simple BMI systems that use power spectral modulation in hand and foot cortex for independent control of 2 degrees of freedom.
\end{abstract}

(http://thejns.org/doi/abs/10.3171/2013.3.FOCUS1373)

KEY WoRDS $\bullet \quad$ stereoelectroencephalography $\bullet$ depth electrode $\bullet$
brain-machine interface $\bullet \quad$ brain-computer interface $\bullet$ epilepsy monitoring unit

I $\mathrm{N}$ a subset of patients with medically intractable focal epilepsy, invasive brain monitoring is recommended to best identify the location of the epileptogenic zone. These patients are admitted to an Epilepsy Monitoring Unit (usually between 3 and 15 days) to await seizure occurrence to better localize the epileptic focus. ${ }^{33}$ Subdural grids are thin flexible sheets or strips of electrodes and are the most commonly used electrodes for invasive epilepsy monitoring in the US. Alternatively, SEEG uses "depth electrodes" consisting of very thin (approximately 1-2

Abbreviations used in this paper: $\mathrm{BMI}=$ brain-machine interface; $\mathrm{EEG}=$ electroencephalography; SEEG = stereo-EEG. $\mathrm{mm}$ ), flexible cylindrical leads with multiple concentric metal electrodes along the length of the lead. Subdural grids require implantation via craniotomy, whereas each SEEG lead only requires a small bur hole placed percutaneously. A stiff wire insertion tool is used to insert the flexible SEEG electrode into the brain to a stereotactically defined depth and angle.

Subdural grid electrodes have the benefit of sampling a large cortical surface area. However, identifying activity in deeper structures, such as the hippocampus, is more difficult with subdural grids. Stereoelectroencephalography electrodes, on the other hand, have the benefit of sampling deeper structures but are limited in terms of cortical 


\section{S. Vadera et al.}

mapping. ${ }^{32}$ Whereas SEEG was originally described by Bancaud et al. in $1965,{ }^{3}$ it has only become increasingly used in the US since 2009.14,32

Brain-machine interfacing is an area of research that is increasingly taking place in tandem with invasive brain monitoring for epilepsy, 1,2,6-8,10,11,18-30,35 even though BMI systems are being developed primarily for paralyzed individuals. Brain-machine interface systems "decode" some aspect of brain processing in real time and use that decoded information to control an assistive device. Motorsystem BMIs decode one's attempted or desired movement from the recorded brain signals. The decoded movement information can then be used in real time to control the movement of a device such as a computer cursor, a wheelchair-mounted robotic arm, or even one's own paralyzed arm reanimated via implanted peripheral nerve stimulators that can activate the paralyzed muscles. ${ }^{36}$ Brain-machine interfaces are also being developed to enable fully paralyzed or "locked-in" individuals to communicate via braindriven typing programs. These communication BMIs often use nonmotor signals, such as changes in brain activity that occur when the letter the person wishes to type flashes on the screen.

A limited number of paralyzed individuals have now been implanted with invasive brain recording electrodes specifically for use in prototype BMI systems. ${ }^{4,5,12,13}$ 15-17,31,34 Patients in the Epilepsy Monitoring Unit provide a unique opportunity to speed up BMI development by providing a pool of research participants that already have invasive brain recording electrodes implanted. Current literature on BMI studies performed in the Epilepsy Monitoring Unit reflects the prevalence of subdural grid electrodes in brain monitoring. ${ }^{1,2,6-8,10,11,19-29,35}$ To date, there have been very few BMI studies conducted using SEEG, ${ }^{18,30}$ and none, to our knowledge, have investigated the feasibility of using SEEG for continuous control of $2 \mathrm{D}$ cursor movements. In this paper we present our initial experiences conducting motor BMI testing with 2 patients who underwent SEEG electrode implantation for localization of ictal onset. Our results show that both foot and hand movements could be detected on a single SEEG electrode lead when recording contacts were located both in the hand knob area and in the foot area. Our findings point to some potential advantages of SEEG electrodes over subdural grids in certain types of BMI systems.

\section{Methods}

\section{Epilepsy Workup and Patient Selection}

Prior to invasive electrode implantation, both patients were discussed in a multidisciplinary epilepsy management conference that included neurosurgeons, epileptologists, radiologists, and neuropsychologists. Standard preoperative workup for epilepsy surgery was performed for both patients, which included high-resolution MRI, magnetoencephalography, SPECT, and PET testing. Patient 1 also underwent preoperative neuropsychological testing based on the location of the lesion. The diagnosis of medically refractory focal epilepsy was made using video-EEG.

After discussion in the epilepsy management confer- ence, both patients were deemed candidates for SEEG electrode implantation because of the deep location of their lesions. The epileptogenic focus in Patient 1 was hypothesized to be located along the left lateral ventricle, where small foci of subependymal gray matter heterotopias were identified. A total of 13 SEEG electrodes with 10-12 contacts each were implanted in the left hemisphere and an additional 2 SEEG electrodes (10 contacts each) were implanted on the right side. Magnetic resonance imaging in Patient 2 suggested a deep lesion in the dorsal surface of the right posterior cingulate, suggestive of cortical dysplasia. A total of 11 SEEG electrodes were implanted in the right hemisphere (10-12 contacts each), and 2 electrodes (10 contacts each) were implanted in the left hemisphere. Electrodes and the insertion stylus were manufactured by Ad-Tech Medical Instrument Corporation.

Decisions on the number of SEEG electrodes to implant and their locations were based solely on clinical diagnostic requirements. The BMI study was not a factor in determining the electrode implantation locations. Patient 1 participated in 2 testing sessions on different days. Patient 2 was only available for 1 testing session. All research-related activities were approved by the Cleveland Clinic Institutional Review Board, and signed consent forms were obtained from both participants.

\section{Methods for 2D Cursor Control}

The Y-shaped splitter connectors were inserted into the SEEG electrode leads, allowing for a copy of the voltage traces to go to our BMI testing equipment while clinical monitoring continued uninterrupted. For this study, 32 SEEG signals were connected to our BMI system via the Y-shaped splitter connectors. However, with Patient 2, 4 of the connectors experienced technical difficulties. Therefore, Patient 1 used all 32 SEEG electrodes for the BMI experiments, but Patient 2 used only 28 SEEG signals. The subset of electrode contacts was chosen based on their proximity to areas of the brain that might convey useful movement information. Figure $1 \mathrm{~A}$ and $\mathrm{B}$ show the approximate SEEG lead locations overlaid on each patient's brain, reconstructed from imaging data; circles indicate the electrode contacts used in the BMI study.

This study used the same testing protocol we have used in previous BMI studies, in which brain signals recorded via EEG electrodes were used to control 2D movements of an object on a screen. ${ }^{9}$ The experimental paradigm and signal processing methods used were similar to those we published in our previous EEG study and are briefly summarized below.

In this study, we were not trying to decode precise limb motion from the associated brain signals. Instead, we used more robust power spectral changes associated with moving or resting 2 different body parts to control the horizontal and vertical components of cursor velocity in real time. Both participants received individual SEEG leads with recording contacts in both the hand knob area and in the foot motor cortex. Patient 2 also had an SEEG lead spanning hand and foot sensory cortex. Therefore, hand and foot movements were chosen for use in this task. Specifically, SEEG power spectral changes reflecting the degree of wiggling compared with resting the hand were 


\section{Brain-machine interfacing with SEEG electrodes}
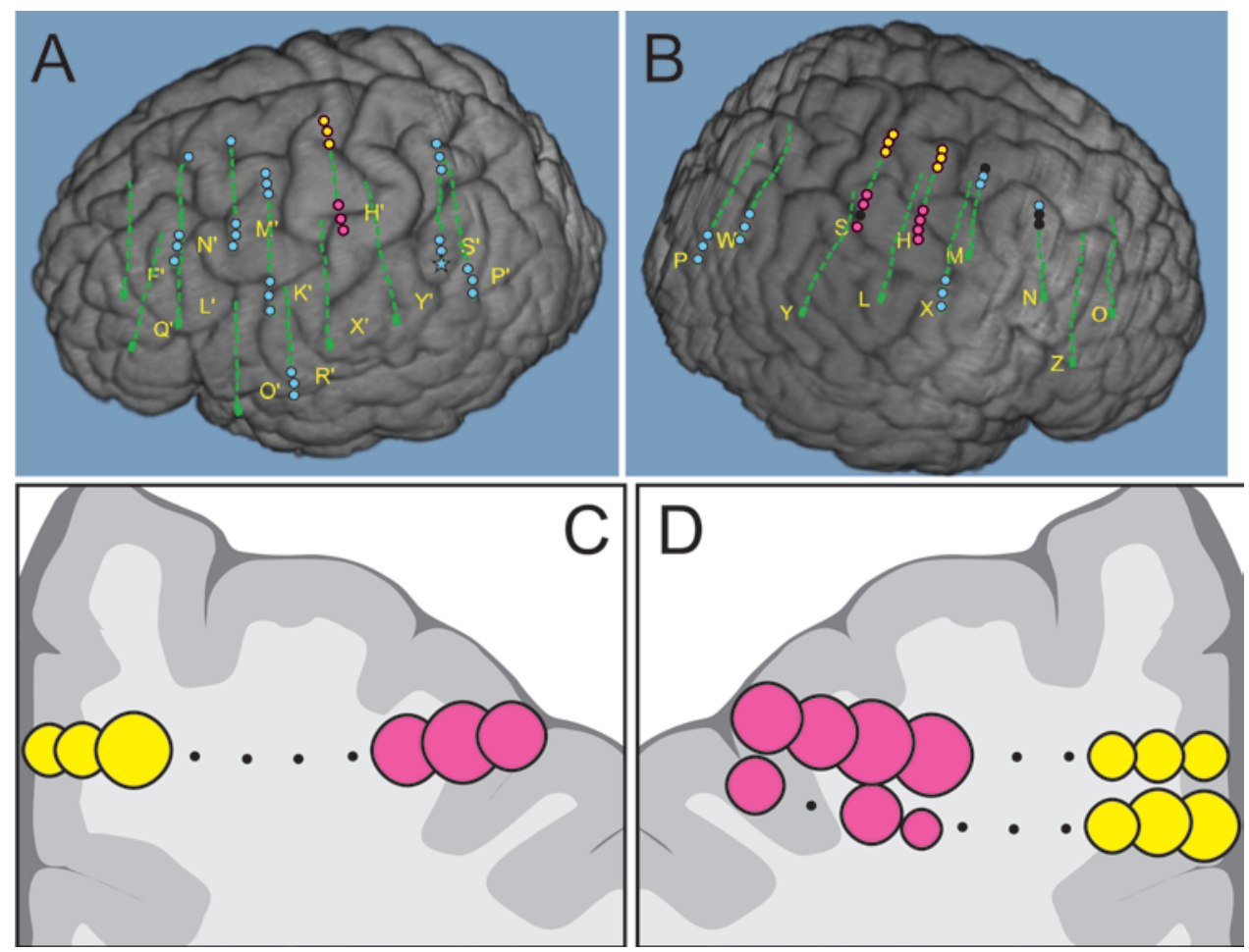

FIG. 1. Stereoelectroencephalography electrode locations in the 2 patients. $\quad A$ and B: Electrode locations for Patients 1 and 2, respectively. Circles indicate contacts that were used for the BMl study. Blue = contacts not significantly modulated with movement; pink = contacts modulated with hand movement; yellow = contacts modulated with foot movement; $b$ lack = contacts excluded due to noise contamination; star = contact outside of the brain. C and D: Coronal section diagrams illustrate the electrode leads traversing hand and foot cortex for Patients 1 and 2, respectively. Circle size indicates the relative accuracy of the hand (pink) or foot (yellow) movement information decoded from each individual contact as measured by the correlation coefficient between the predicted and actual hand or foot movement state. Scaling ranges from $r=0.56$ (largest circle) to $r=0.27$ (smallest circle excluding dots).

assigned to control the horizontal component of the cursor velocity. Power spectral changes reflecting the degree of wiggling compared with resting the foot were assigned to control the vertical component of the cursor velocity in real time.

To generate a decoding function to translate each participant's brain activity into $2 \mathrm{D}$ cursor motion in real time, we first collected 3 minutes of baseline data in which the participants wiggled/rested their hands and feet on cue. To cue the participants, 4 targets were presented on the screen, 1 at a time. The participants were instructed to wiggle or rest their hands or feet in the 4 different combinations that would be required to move the cursor to each target during the upcoming brain-control task. This process also served to acclimate the participants to performing the different combinations of hand and foot movements. Figure 2 illustrates the 4 movement combinations as well as how moving and/or resting the hand and foot by different degrees would be used to move the brain-controlled cursor during the closed-loop target acquisition task.

The collected baseline data were used to generate a decoding function by first using a common spatial pattern algorithm to generate 8 linear combinations of the raw signals that maximized the difference in magnitude between the moving and resting states for the hand and foot (algorithm described in Foldes and Taylor ${ }^{9}$ ). Power spectral data from the original raw SEEG signals along with the 8 new combined signals were then calculated. $\mathrm{X}$ and $\mathrm{Y}$ movement decoding functions were generated by regressing the power values against the $\mathrm{X}$ and $\mathrm{Y}$ components of the 4 target locations used to cue the different hand and foot movements. Power values from only 4 frequency bands were used on Patient 1's first day of testing $(0-0.75 \mathrm{~Hz}, 0.75-2.25 \mathrm{~Hz}, 2.25-9 \mathrm{~Hz}$, and 9-30 $\mathrm{Hz}$ ), but power values in 7 frequency bands were used for Patient 1's second testing session and for Patient 2's testing session $(4.5-7.5 \mathrm{~Hz}, 7.5-10.5 \mathrm{~Hz}, 10.5-13.5 \mathrm{~Hz}$, $13.5-18 \mathrm{~Hz}, 18-22.5 \mathrm{~Hz}, 22.5-36 \mathrm{~Hz}$, and $36-100.5 \mathrm{~Hz}$ ). The regression coefficients became the decoding function that would map each participant's power values to an $\mathrm{X}$ and $\mathrm{Y}$ cursor velocity at each time step in the subsequent cursor control task.

Once each patient's decoder coefficients were generated, the task was switched to the 2D target acquisition task in which participants had to use their decoded brain signals to move a cursor to the displayed targets in real time. At the beginning of each trial, the cursor would appear in the center of the screen along with 1 of the 4 radial targets. Participants had up to 5 seconds to use their brain signals to steer the cursor to the target before the cursor would be recentered, and a new target would appear. The participants were instructed to wiggle their hands when they needed to steer the cursor right and to rest their hands 

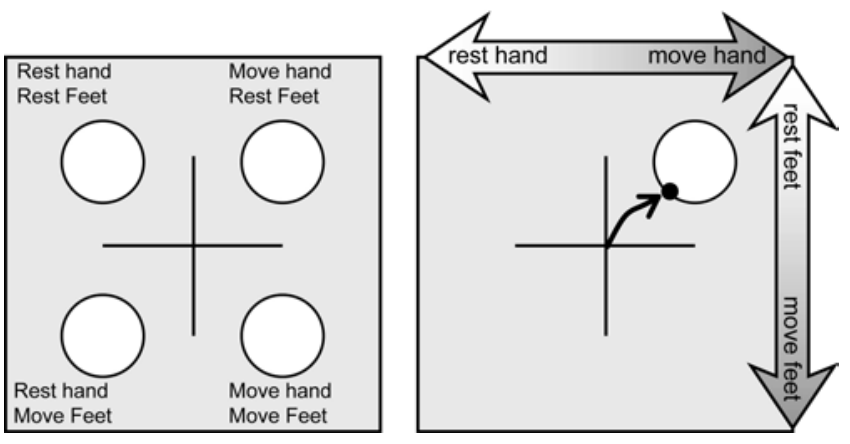

FIG. 2. Illustration of the hand and foot actions used for $2 \mathrm{D}$ cursor control. Left: During baseline data collection, participants performed the 4 listed combinations of hand and foot movements when each target appeared. Right: During the brain-controlled cursor task, participants had to vary how strongly they wiggled or rested their hand or foot to steer the cursor from the center of the workspace to the displayed target. In the example shown, the participant would have wiggled his hand and relaxed his foot to cause the cursor to move to the target.

when they needed to steer the cursor left; similarly they were instructed to wiggle their feet when they needed to steer the cursor down and rest their feet when they needed to steer the cursor up (Fig. 2). At the start of each session, the targets were very large so the participants did not have to move the cursor very far to hit the target. However, as performance improved, the task difficulty was increased by automatically reducing the target size.

\section{Additional Post Hoc Analysis}

Additional analyses were performed after the testing sessions were over to determine which electrode contacts were most useful for movement control and to determine if cursor control could have been improved had we eliminated nonuseful electrode contacts from the decoder. We also estimated how accurately 2D movement could have been controlled had we used 1 or 2 electrode leads passing through both the hand and foot cortical areas.

The usefulness of each individual electrode contact was determined by calculating how well the signal from that contact alone could be decoded into the movements needed to reach the target. Data from the brain-control task were used for building new decoding functions offline for each individual electrode. Individual electrode decoding functions were generated in a manner similar to the original full decoding function except that only power bands from 1 electrode contact at a time were included in the regression. Because the cursor was moving during the brain-control task, the regression was performed between the power from each individual contact and the movement needed at each time step to move the cursor to the target from its current position.

To ensure accurate assessment of the decoding performance of each individual electrode contact, a 10-fold cross-validation process was used in which $90 \%$ of the time points from the brain-control task formed the training data set that was used to build the individual decoding functions. The remaining $10 \%$ of the data (testing set) was used to determine the accuracy of the decoders by calculating the correlation between the actual movement needed at each time step and the movement predicted by each contact's decoding function. This process was then repeated 10 times, each time using a different 10\%/90\% of the data for testing/training, respectively.

Not surprisingly, the only electrode contacts within the brain that were significantly modulated with hand and foot movements were those contacts directly in hand or foot motor and/or sensory cortex (pink and yellow circles in Fig. 1). Figure $1 C$ and $\mathrm{D}$ illustrate these electrodes from a coronal view in which the circle size indicates how well each individual contact could decode hand (pink) or foot (yellow) movements.

The entire data set from the brain-control task was then used to build the new decoding functions that tested how well 2D movements could be controlled if only the 1 or 2 SEEG leads spanning the hand and foot areas were used for control. Those new refined decoders were then applied to the original baseline data set during which participants just wiggled or rested their hands and feet on cue. We chose to build our new decoding functions on the brain-control data set and apply it to the baseline data set for two reasons. First, the brain control data set was much larger, which should, in itself, improve the accuracy of the decoding functions built from it. Second, the baseline data set consisted of uniform segments of data in which participants were consistently performing the combinations of hand and foot wiggles and resting needed to move directly to each of the 4 targets. Therefore, perfectly decoded movement trajectories from the baseline data set should point directly toward the intended targets, thus making it easy to visualize and quantify the offline movement accuracy.

\section{Results}

Figure 3 shows the cursor trajectories generated when the patients used their decoded brain signals to control the cursor in real time (plots labeled "closedloop") as well as additional theoretical trajectories calculated offline using the signals recorded on only 1 or 2 SEEG leads spanning the hand/foot cortex (plots labeled "open-loop"). The number of electrode contacts used for decoding in each plot is listed below the "closed-loop" and "open-loop" labels. Because it is difficult to clearly see all the overlapping trajectories, trajectories are also replotted in an exploded view underneath each primary plot. These exploded views separate out trajectories to each target, making it easier to see the spread and consistency of movements to each target. The black lines in the exploded views span the center starting position and the center of the outer target positions. Similarly the thicker, straight colored lines go from the center start position to the target centers in the normal trajectory plots. Therefore, perfectly decoded trajectories should be in line with each black line in the exploded view and in line with each thick, straight colored line in the standard view.

Although neither participant performed particularly well during closed-loop control, the exploded view plots show that trajectories to most targets at least went toward the correct half of the workspace and were therefore not random. Notice how Patient 1's trajectories were most accurate in the $\mathrm{X}$ direction on the first day and most accurate in the Y direction on the second day (Fig. 3). However, the 
Brain-machine interfacing with SEEG electrodes

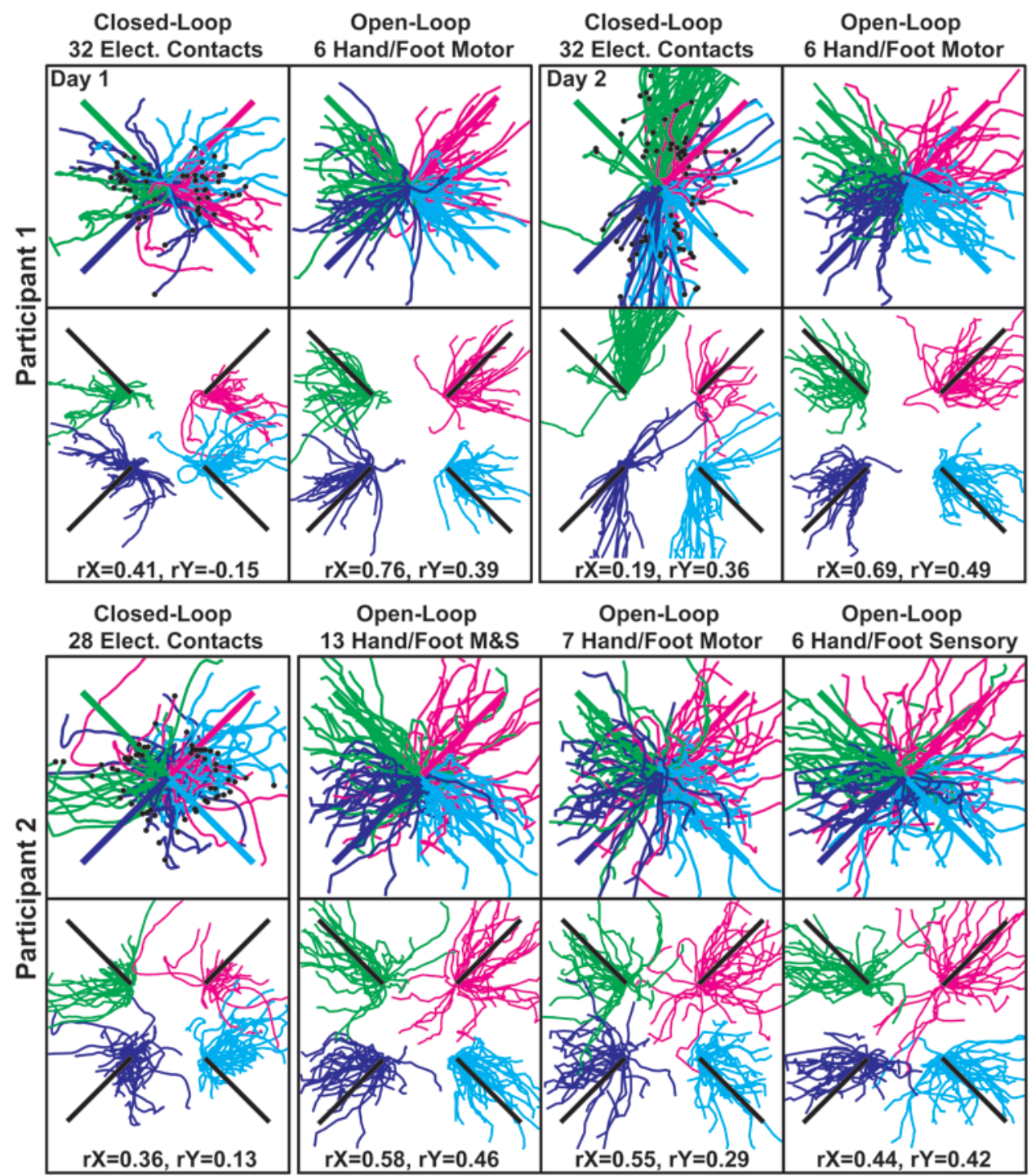

FIG. 3. Two-dimensional cursor trajectories generated during the brain-control task (closed-loop) and calculated offline from the baseline data using only 1 or 2 SEEG leads spanning hand and foot cortex (open-loop; 13 contacts for 2 leads and 6 or 7 contacts for 1 lead). Trajectories are color coded by their intended targets. Black dots in the closed-loop plots indicate when the correct target was hit. The upper row of plots for each participant shows the trajectories plotted normally. Those same trajectories are replotted directly underneath with the trajectories for each target separated for easier viewing. The $r X$ and $r Y$ numbers listed below each plot indicate the correlation coefficients between the actual $X$ and $Y$ movements needed and the movement decoded from the neural signals at each time step. Elect. = electrode; $M \& S=$ motor and sensory.

offline trajectories generated from the 1 SEEG lead spanning the hand and foot regions were fairly good and fairly similar between days. These results suggest the inclusion of nonuseful electrode contacts during closed-loop control likely degraded the decoded movement command with unrelated noise. The decoders built on different days handled this unrelated noise differently, resulting in different problems with closed-loop control on different days.

Note that the exploded views of the closed-loop trajectories do not show any trajectories that make it out to the distal ends of the black lines, even though open-loop trajectories do tend to move outward around each black line. During closed-loop control, the movement trial ended as soon as the cursor hit the target. Therefore, closedloop trajectories that successfully hit the target were trun- cated at the target boundary. However, trajectories that missed the targets continued to grow until either the trial ended or the person was able to redirect the trajectory back to the target. This truncation of the good trajectories but not the bad trajectories should be kept in mind when assessing the spread of the closed-loop trajectories. For the closed-loop trajectories, the $\mathrm{rX}$ and $\mathrm{rY}$ values listed (Fig. 3) are the correlations between the movement vectors that were actually generated and the direction vectors needed to move the cursor toward the target at each time step. Because of this truncation of good movement trials, there was a disproportionately large number of time steps from missed trials compared with good trials in these closed-loop correlation calculations. For closed-loop control, these correlation values are not a true measure of 


\section{S. Vadera et al.}

movement accuracy as in the open-loop plots, in which all data were included in the rX and rY calculations and no truncation took place. For the offline data, all correlations were significant at $\mathrm{p}<0.00001$.

From the exploded trajectory plots, one can see that most trajectories calculated offline generally went in the correct direction with some degree of spread. In most cases, the spread stayed within the correct quadrant of the workspace. Because those trajectories were generated offline, no corrective movements could be made to redirect the cursor back toward the hypothetical target. The offline decoding accuracy values observed here with single SEEG electrode leads are consistent with those from our EEG-BMI studies in which participants used 32 scalp EEG electrodes for similar open- and closedloop 2D cursor control tasks. The closed-loop example given in Foldes and Taylor ${ }^{9}$ suggests that the quality of offline decoding noted in this study can result in reasonably functional 2D cursor movements when the person has visual feedback and can make corrective movements online if a trajectory starts to drift off course.

Patient 2 had 2 leads that spanned hand and foot cortex: 1 lead was in the motor area and 1 was in the sensory area. Trajectories were generated offline using just the single SEEG hand/foot motor lead (7 contacts), using just the single hand/foot sensory lead (6 working contacts), and then using both the motor and sensory leads together (13 contacts). While offline movement accuracy was better when both motor and sensory leads were used, the increase in performance was small over either one alone. For paralyzed individuals, motor areas may be more easily modulated with attempted movements than sensory areas, although both have been shown to be useful in EEG studies.

\section{Discussion}

Patients undergoing invasive electrode monitoring for medically intractable focal epilepsy offer a unique opportunity to assess the benefits and drawbacks of a variety of BMI systems. Because the majority of patients in the US undergoing invasive testing for epilepsy are implanted with subdural grid electrodes, there is a great deal of literature and research related to subdural grid-based BMI systems. ${ }^{1,2,6-8,10,11,19-29,35}$ On the other hand, there is a relative dearth of SEEG-related BMI studies..$^{18,30}$

Previous SEEG BMI studies have demonstrated the possibility of using SEEG electrodes in communication BMIs in which letters were selected by involuntary brain responses. ${ }^{18,30}$ This study, for the first time, demonstrated the use of SEEG electrodes for continuous 2D motion control using voluntary activity in hand and foot cortical areas. Trajectories generated during the real-time cursor control task were more accurate than one would expect by chance. However, further post hoc analysis suggested that better directional control could have been achieved by including only the electrode contacts that were directly in the hand and foot motor areas and excluding contacts conveying unrelated activity.

Most notably, the only electrode contacts within the brain that were significantly modulated with hand/foot movements were all located on the same 1 or 2 SEEG leads, suggesting that BMI control of 2D movements could be achieved with electrode leads implanted through just 1 or 2 bur holes. Plots from Patient 2 in Fig. 3 showed the relative difference between using 1 versus 2 leads for control. Although there is some improvement with 2 electrodes, 1 lead may be adequate as BMI users learn to modulate their brain areas more effectively with practice.

The circle sizes in Fig. 1C and D show that 3-4 different SEEG contacts within each hand or foot cortical area were all generating similarly useful signals for control. These similarities suggest that the implantation depth within the various cortical layers does not have to be very precise because there is a large range in which electrode contacts can be placed for good BMI signals to be acquired. Furthermore, with BMIs that use scalp surface EEGs, the left and right foot movements cannot be differentiated because the current from both hemispheres blends together to form 1 combined generic "foot" signal at the top of the head. Stereoelectroencephalography electrodes could potentially be placed bilaterally, acquiring separate signals from both left versus right hand and left versus right foot regions. Bilateral implants could theoretically provide 4 relatively independent control signals for more complex BMI applications.

\section{Conclusions}

Stereoelectroencephalography electrodes for BMIs have many of the same pros and cons as when using SEEG electrodes in epilepsy monitoring. Stereoelectroencephalography electrodes allow us to reach deep structures, such as the foot motor cortex, which was shown to be useful for cursor control in this study. Stereoelectroencephalography electrodes also allowed us to reach both superficial and deep structures with the same lead and could be implanted percutaneously through small bur holes instead of the larger craniotomies required by subdural grid electrodes. This study demonstrated the feasibility of performing 2D continuous movement control using only a single SEEG electrode lead. These results warrant further exploration of SEEG electrode technology for BMI applications.

\section{Disclosure}

This work was supported by the National Institute of Neurological Disorders and Stroke, NIH (grant no. R01-NS058871), the Department of Veterans Affairs (Merit Review no. B4195R), the Cleveland Clinic, and Case Western Reserve University.

Author contributions to the study and manuscript preparation include the following. Conception and design: Taylor. Acquisition of data: Taylor, Marathe. Analysis and interpretation of data: Taylor, Vadera. Drafting the article: Taylor, Vadera. Critically revising the article: all authors. Reviewed submitted version of manuscript: all authors. Approved the final version of the manuscript on behalf of all authors: Taylor. Statistical analysis: Taylor. Administrative/technical/material support: Taylor, Marathe, Gonzalez-Martinez. Study supervision: Taylor.

\section{References}

1. Acharya S, Fifer MS, Benz HL, Crone NE, Thakor NV: Electrocorticographic amplitude predicts finger positions during 
slow grasping motions of the hand. J Neural Eng 7:046002, 2010

2. Anderson NR, Blakely T, Schalk G, Leuthardt EC, Moran DW: Electrocorticographic (ECoG) correlates of human arm movements. Exp Brain Res 223:1-10, 2012

3. Bancaud J, Talairach J, Bonis A, Schaub C, Szikla G, Morel P, et al: La Stéréo-Electroencéphalographie dans l'Epilepsie. Paris: Masson et Cie, 1965

4. Chadwick EK, Blana D, Simeral JD, Lambrecht J, Kim SP, Cornwell AS, et al: Continuous neuronal ensemble control of simulated arm reaching by a human with tetraplegia. J Neural Eng 8:034003, 2011

5. Collinger JL, Wodlinger B, Downey JE, Wang W, Tyler-Kabara EC, Weber DJ, et al: High-performance neuroprosthetic control by an individual with tetraplegia. Lancet 381:557564,2013

6. Felton EA, Wilson JA, Williams JC, Garell PC: Electrocorticographically controlled brain-computer interfaces using motor and sensory imagery in patients with temporary subdural electrode implants. Report of four cases. J Neurosurg 106: 495-500, 2007

7. Fifer MS, Acharya S, Benz HL, Mollazadeh M, Crone NE, Thakor NV: Toward electrocorticographic control of a dexterous upper limb prosthesis: building brain-machine interfaces. IEEE Pulse 3:38-42, 2012

8. Flamary R, Rakotomamonjy A: Decoding finger movements from ECoG signals using switching linear models. Front Neurosci 6:29, 2012

9. Foldes ST, Taylor DM: Offline comparison of spatial filters for two-dimensional movement control with noninvasive field potentials. J Neural Eng 8:046022, 2011

10. Gunduz A, Sanchez JC, Carney PR, Principe JC: Mapping broadband electrocorticographic recordings to two-dimensional hand trajectories in humans motor control features. Neural Netw 22:1257-1270, 2009

11. Hermes D, Siero JC, Aarnoutse EJ, Leijten FS, Petridou N, Ramsey NF: Dissociation between neuronal activity in sensorimotor cortex and hand movement revealed as a function of movement rate. J Neurosci 32:9736-9744, 2012

12. Hochberg LR, Bacher D, Jarosiewicz B, Masse NY, Simeral JD, Vogel J, et al: Reach and grasp by people with tetraplegia using a neurally controlled robotic arm. Nature 485:372-375, 2012

13. Hochberg LR, Serruya MD, Friehs GM, Mukand JA, Saleh M, Caplan AH, et al: Neuronal ensemble control of prosthetic devices by a human with tetraplegia. Nature 442:164-171, 2006

14. Kahane P, Landré E, Minotti L, Francione S, Ryvlin P: The Bancaud and Talairach view on the epileptogenic zone: a working hypothesis. Epileptic Disord 8 (Suppl 2):S16-S26, 2006

15. Kennedy P, Andreasen D, Ehirim P, King B, Kirby T, Mao $\mathrm{H}$, et al: Using human extra-cortical local field potentials to control a switch. J Neural Eng 1:72-77, 2004

16. Kim SP, Simeral JD, Hochberg LR, Donoghue JP, Black MJ: Neural control of computer cursor velocity by decoding motor cortical spiking activity in humans with tetraplegia. J Neural Eng 5:455-476, 2008

17. Kim SP, Simeral JD, Hochberg LR, Donoghue JP, Friehs GM, Black MJ: Point-and-click cursor control with an intracortical neural interface system by humans with tetraplegia. IEEE Trans Neural Syst Rehabil Eng 19:193-203, 2011

18. Krusienski DJ, Shih JJ: Control of a brain-computer interface using stereotactic depth electrodes in and adjacent to the hippocampus. J Neural Eng 8:025006, 2011

19. Kubánek J, Miller KJ, Ojemann JG, Wolpaw JR, Schalk G: Decoding flexion of individual fingers using electrocorticographic signals in humans. J Neural Eng 6:066001, 2009

20. Leuthardt EC, Schalk G, Wolpaw JR, Ojemann JG, Moran
DW: A brain-computer interface using electrocorticographic signals in humans. J Neural Eng 1:63-71, 2004

21. Milekovic T, Ball T, Schulze-Bonhage A, Aertsen A, Mehring C: Error-related electrocorticographic activity in humans during continuous movements. J Neural Eng 9:026007, 2012

22. Milekovic T, Fischer J, Pistohl T, Ruescher J, Schulze-Bonhage A, Aertsen A, et al: An online brain-machine interface using decoding of movement direction from the human electrocorticogram. J Neural Eng 9:046003, 2012

23. Miller KJ, Hermes D, Honey CJ, Hebb AO, Ramsey NF, Knight RT, et al: Human motor cortical activity is selectively phase-entrained on underlying rhythms. PLOS Comput Biol 8:e1002655, 2012

24. Miller KJ, Zanos S, Fetz EE, den Nijs M, Ojemann JG: Decoupling the cortical power spectrum reveals real-time representation of individual finger movements in humans. J Neurosci 29:3132-3137, 2009

25. Pistohl T, Ball T, Schulze-Bonhage A, Aertsen A, Mehring C: Prediction of arm movement trajectories from ECoG-recordings in humans. J Neurosci Methods 167:105-114, 2008

26. Pistohl T, Schulze-Bonhage A, Aertsen A, Mehring C, Ball T: Decoding natural grasp types from human ECoG. Neuroimage 59:248-260, 2012

27. Reddy CG, Reddy GG, Kawasaki H, Oya H, Miller LE, Howard MA III: Decoding movement-related cortical potentials from electrocorticography. Neurosurg Focus 27(1):E11, 2009

28. Schalk G, Kubánek J, Miller KJ, Anderson NR, Leuthardt EC, Ojemann JG, et al: Decoding two-dimensional movement trajectories using electrocorticographic signals in humans. J Neural Eng 4:264-275, 2007

29. Scherer R, Zanos SP, Miller KJ, Rao RP, Ojemann JG: Classification of contralateral and ipsilateral finger movements for electrocorticographic brain-computer interfaces. Neurosurg Focus 27(1):E12, 2009

30. Shih JJ, Krusienski DJ: Signals from intraventricular depth electrodes can control a brain-computer interface. J Neurosci Methods 203:311-314, 2012

31. Simeral JD, Kim SP, Black MJ, Donoghue JP, Hochberg LR: Neural control of cursor trajectory and click by a human with tetraplegia 1000 days after implant of an intracortical microelectrode array. J Neural Eng 8:025027, 2011

32. Vadera S, Mullin J, Bulacio J, Najm I, Bingaman W, Gonzalez-Martinez J: SEEG following subdural grid placement for difficult to localize epilepsy. Neurosurgery [epub ahead of print], 2013

33. Van Gompel JJ, Stead SM, Giannini C, Meyer FB, Marsh WR, Fountain T, et al: Phase I trial: safety and feasibility of intracranial electroencephalography using hybrid subdural electrodes containing macro- and microelectrode arrays. Neurosurg Focus 25(3):E23, 2008

34. Wang W, Collinger JL, Degenhart AD, Tyler-Kabara EC, Schwartz AB, Moran DW, et al: An electrocorticographic brain interface in an individual with tetraplegia. PLoS ONE 8:e55344, 2013

35. Wang Z, Ji Q, Miller KJ, Schalk G: Prior knowledge improves decoding of finger flexion from electrocorticographic signals. Front Neurosci 5:127, 2011

36. Yu DT, Kirsch RF, Bryden AM, Memberg WD, Acosta AM: A neuroprosthesis for high tetraplegia. J Spinal Cord Med 24: 109-113, 2001

Manuscript submitted February 18, 2013.

Accepted March 12, 2013.

Please include this information when citing this paper: DOI: 10.3171/2013.3.FOCUS1373.

Address correspondence to: Dawn M. Taylor, Ph.D., The Cleveland Clinic, Lerner Research Institute, 9500 Euclid Avenue, NC30, Cleveland, Ohio 44195. email: dxt42@case.edu. 